UFIFT-QG-12-07

\title{
Representing the Vacuum Polarization on de Sitter
}

\author{
Katie E. Leonard ${ }^{1 *}$, Tomislav Prokopec ${ }^{2 \dagger}$ and Richard P. Woodard ${ }^{1, \ddagger}$ \\ ${ }^{1}$ Department of Physics, University of Florida \\ Gainesville, FL 32611, UNITED STATES \\ 2 Institute of Theoretical Physics (ITP) \& Spinoza Institute, \\ Utrecht University, Postbus 80195, 3508 TD Utrecht, \\ THE NETHERLANDS
}

\begin{abstract}
Previous studies of the vacuum polarization on de Sitter have demonstrated that there is a simple, noncovariant representation of it in which the physics is transparent. There is also a cumbersome, covariant representation in which the physics is obscure. Despite being unwieldy, the latter form has a powerful appeal for those who are concerned about de Sitter invariance. We show that nothing is lost by employing the simple, noncovariant representation because there is a closed form procedure for converting its structure functions to those of the covariant representation. We also present a vastly improved technique for reading off the noncovariant structure functions from the primitive diagrams. And we discuss the issue of representing the vacuum polarization for a general metric background.
\end{abstract}

PACS numbers: 04.62.+v, 98.80.Cq, 12.20.Ds

\footnotetext{
* e-mail: katie@phys.ufl.edu

† e-mail: T.Prokopec@uu.nl

‡e-mail: woodard@phys.ufl.edu
} 


\section{Introduction}

The vacuum polarization $i\left[{ }^{\mu} \Pi^{\nu}\right]\left(x ; x^{\prime}\right)$ is a bi-vector density which can be used to quantum-correct the classical Maxwell equations,

$$
\partial_{\nu}\left[\sqrt{-g} g^{\nu \rho} g^{\mu \sigma} F_{\rho \sigma}(x)\right]+\int d^{4} x^{\prime}\left[{ }^{\mu} \Pi^{\nu}\right]\left(x ; x^{\prime}\right) A_{\nu}\left(x^{\prime}\right)=J^{\mu}(x) .
$$

The vacuum polarization encodes two sorts of information:

- How quantum 0-point fluctuations affect photons; and

- How quantum 0-point fluctuations modify the electromagnetic response to charges and currents.

In flat space background, neither charged matter nor gravitons have any effect on photons, and significant modifications to the electromagnetic response are limited to the immediate vicinity of the source [1, 2]. An analogous local effect occurs for gravitons [3]. Things can be very different in de Sitter background. The inflationary production of light, minimally coupled, charged scalars induces so much vacuum polarization that the photon develops a mass [4, 5, 6, 7] and electromagnetic forces suffer comparable changes [8]. A study of the effect of inflationary gravitons is far advanced [10]. Although they cannot induce a photon mass, it is expected that buffeting by the vast ensemble of infrared gravitons will lead to a progressive growth of the field strength. This occurs for massless fermions [11, 12, 13], and probably also for massive ones [14], owing to the spin-spin coupling [15], which photons also possess.

In view of its physical importance, consideration should clearly be given to the best way of representing the tensor structure of $\left.i^{[\mu} \Pi^{\nu}\right]\left(x ; x^{\prime}\right)$. It is transverse on each index, and also symmetric under interchange,

$$
i\left[{ }^{\mu} \Pi^{\nu}\right]\left(x ; x^{\prime}\right)=i\left[{ }^{\nu} \Pi^{\mu}\right]\left(x^{\prime} ; x\right) .
$$

These two facts mean $i\left[{ }^{\mu} \Pi^{\nu}\right]\left(x ; x^{\prime}\right)$ can have at most $\frac{1}{2} \times 4 \times 5-4=6$ independent components. However, on flat space background the vacuum polarization can be represented using only a single structure function,

$$
i\left[{ }^{\mu} \Pi_{\text {flat }}^{\nu}\right]\left(x ; x^{\prime}\right)=-\left[\eta^{\mu \nu} \partial^{2}-\partial^{\mu} \partial^{\nu}\right] \Pi\left(\Delta x^{2}\right),
$$


where $\eta^{\mu \nu}$ is the spacelike Minkowski metric and $\Delta x^{2} \equiv\left\|\vec{x}-\vec{x}^{\prime}\right\|^{2}-\left(\mid x^{0}-\right.$ \left.${x^{\prime}}^{0} \mid-i \epsilon\right)^{2}$.

On the homogeneous and isotropic backgrounds of cosmology the vacuum polarization can be written as a linear combination of four algebraically independent tensors [16]. Transversality provides two differential relations between their coefficients, so there should be two independent structure functions [16] in a general cosmological background. The earliest de Sitter computations [4, 5, 7] were made in conformal coordinates,

$$
d s^{2}=a^{2}\left(-d \eta^{2}+d \vec{x} \cdot d \vec{x}\right) \quad, \quad a(\eta)=-\frac{1}{H \eta},
$$

where $H$ is the Hubble constant. These early works represented the vacuum polarization as,

$i\left[{ }^{\mu} \Pi^{\nu}\right]\left(x ; x^{\prime}\right)=\left[\eta^{\mu \nu} \eta^{\rho \sigma}-\eta^{\mu \sigma} \eta^{\nu \rho}\right] \partial_{\rho} \partial_{\sigma}^{\prime} F\left(x ; x^{\prime}\right)+\left[\bar{\eta}^{\mu \nu} \bar{\eta}^{\rho \sigma}-\bar{\eta}^{\mu \sigma} \bar{\eta}^{\nu \rho}\right] \partial_{\rho} \partial_{\sigma}^{\prime} G\left(x ; x^{\prime}\right)$,

where $\bar{\eta}^{\mu \nu} \equiv \eta^{\mu \nu}+\delta_{0}^{\mu} \delta_{0}^{\nu}$ is the purely spatial part of the Minkowski metric.

The structure functions $F\left(x ; x^{\prime}\right)$ and $G\left(x ; x^{\prime}\right)$ have a simple interpretation in terms of the electric and magnetic susceptibilities $\chi_{e}$ and $\chi_{m}$ [17],

$$
\chi_{e}\left(x ; x^{\prime}\right)=-i F\left(x ; x^{\prime}\right) \quad, \quad \frac{\chi_{m}}{1+\chi_{m}}\left(x ; x^{\prime}\right)=-i G\left(x ; x^{\prime}\right) .
$$

These susceptibilities can be used to obtain the polarization and the magnetization vectors, and the corresponding macroscopic fields, the same as for flat space electrodynamics in a medium,

$$
\begin{array}{lll}
\vec{P}=\chi_{e} \otimes \vec{E} & \Longrightarrow & \vec{D}=\vec{E}+\vec{P}, \\
\vec{M}=\chi_{m} \otimes \vec{B} & \Longrightarrow & \vec{H}=\vec{B}-\vec{M}
\end{array}
$$

Here $\otimes$ signifies convolution, meaning that relations (7-8) are actually nonlocal and can involve integrations within the past light-cone.

Although the original representation (5) has a transparent physical interpretation, and is easy to use in the quantum-corrected Maxwell equations [6], its structure functions $F\left(x ; x^{\prime}\right)$ and $G\left(x ; x^{\prime}\right)$ are not bi-scalar densities because $\eta^{\mu \nu}$ and $\bar{\eta}^{\mu \nu}$ are not bi-tensors. This may appear disturbing to those who believe that the de Sitter group should play the same role in organizing quantum field theory on de Sitter background that the Poincaré group does 
on flat space [18, 19]. It was difficult to form an opinion as to the merit of this view as long as only the noncovariant representation (5) had been studied. Therefore, we recently recast the old results of SQED (scalar quantum electrodynamics) [4, 5, 7] in a covariant form [9],

$$
\begin{aligned}
\left.i{ }^{\mu} \Pi^{\nu}\right]\left(x ; x^{\prime}\right)=\sqrt{-g} \sqrt{-g^{\prime}} D_{\rho} D_{\sigma}^{\prime}\{ & D^{\mu} D^{\prime[\nu} y D^{\prime \sigma]} D^{\rho} y \times f_{1}(y, u, v) \\
& \left.+D^{[\mu} y D^{\rho]} D^{\prime \nu} y D^{\prime \sigma]} y \times f_{2}(y, u, v)\right\} .
\end{aligned}
$$

Here $D^{\rho}$ and $D^{\prime \sigma}$ stand for covariant derivative operators, square brackets denote anti-symmetrization, and the variables $y, u$ and $v$ are,

$$
y\left(x ; x^{\prime}\right)=a a^{\prime} H^{2} \Delta x^{2} \quad, \quad u\left(x ; x^{\prime}\right)=\ln \left(a a^{\prime}\right) \quad, \quad v\left(x ; x^{\prime}\right)=\ln \left(\frac{a}{a^{\prime}}\right) .
$$

The de Sitter invariant length function $y\left(x ; x^{\prime}\right)$ in (9) is related to the geodetic length $\ell\left(x ; x^{\prime}\right)$ from $x^{\mu}$ to $x^{\prime \mu}$ by $y=4 \sin ^{2}\left(\frac{1}{2} H \ell\right)$. When the vacuum polarization is de Sitter invariant, the structure functions are independent of $u$ and $v$, and only one structure function is required, just as in flat space. This happens for the case of a scalar with positive mass-squared [7]. Dependence upon the de Sitter breaking terms $u\left(x ; x^{\prime}\right)$ and $v\left(x ; x^{\prime}\right)$ arises from de Sitter breaking in the massless, minimally coupled scalar propagator [20, 21, 22].

The new representation (9) is not very illuminating, even in the massive case for which it is fully de Sitter invariant 9. Renormalization is unbelievably complicated. (This is also the case for the closely related graviton self-energy [23.) The worst problem is that the correction for dynamical photons - which simply degenerates to a local Proca term in the noncovariant representation [7] - takes the form of a surface term at the initial time in the covariant representation. (This again happens for quantum corrections to dynamical gravitons [24].) We are used to such surface terms being suppressed by powers of the scale factor [21, 22, and sometimes being absorbable into corrections of the initial state [25], so it is unsettling to have them dominate local physics in the de Sitter covariant formulation. We successfully resolved the apparent paradox [9], but the covariant representation (9) seems to be a cumbersome and nearly useless way of describing the vacuum polarization on de Sitter.

There is no reason to expect the covariant representation (9) to be any more useful or revealing for the coming graviton contribution to the vacuum 
polarization [10]. Nor can it be de Sitter invariant. Just as in flat space background [2], the primitive one loop diagrams involve photon and graviton propagators. The photon propagator is manifestly de Sitter invariant in a de Sitter invariant gauge [26], but the graviton propagator must break de Sitter invariance [27, 28, 29, 30, 31]. Of course this de Sitter breaking is the reason why there can be a secular enhancement of the field strength, so it should not be viewed as an irritant but rather as the signal of the inflationary graviton production which motivates the computation.

For all these reasons it seems reasonable to represent the primitive diagrams using the noncovariant representation (5). However, mathematically minded literature continue to be powerfully drawn to de Sitter invariance [18, 19], and one might expect them to prefer the covariant representation (9). The purpose of this paper is to demonstrate that nothing is lost by employing the noncovariant representation (5). We will derive a closed form procedure for converting the noncovariant structure functions $F\left(x ; x^{\prime}\right)$ and $G\left(x ; x^{\prime}\right)$ into the structure functions $f_{1}(y, u, v)$ and $f_{2}(y, u, v)$ of the covariant representation (9). Although the derivation is highly nontrivial, the final result is quite simple, so mathematical physicists who prefer the covariant representation can easily convert to it.

Section 2 reviews some important results which are employed in the analysis. The derivation is made in section 3. In section 4 we check the final transformation formulae using the vacuum polarization of SQED for which the structure functions of both representations are known. We also give a major simplification in the procedure for inferring the noncovariant structure functions from primitive diagrams. Our conclusions comprise section 5. After summarizing results we discuss the fascinating issue of how to represent the vacuum polarization on a general metric background.

\section{Known Results on Tensor Structures}

In this section we review some recently derived result that are necessary for the derivation of section 3 . The first of these results consists of expanding the Minkowski metric and its spatial restriction in terms of covariant derivatives 
of $y\left(x ; x^{\prime}\right)$ and $u\left(x ; x^{\prime}\right)$ [30, 32],

$$
\begin{aligned}
\eta^{\mu \nu} & =\frac{a a^{\prime}}{2 H^{2}}\left\{-D^{\mu} D^{\prime \nu} y+D^{\mu} y D^{\prime \nu} u+D^{\mu} u D^{\prime \nu} y-y D^{\mu} u D^{\prime \nu} u\right\}, \\
\bar{\eta}^{\mu \nu} & =\frac{a a^{\prime}}{2 H^{2}}\left\{-D^{\mu} D^{\prime \nu} y+D^{\mu} y D^{\prime \nu} u+D^{\mu} u D^{\prime \nu} y+(2-y) D^{\mu} u D^{\prime \nu} u\right\} .
\end{aligned}
$$

(Note that covariant derivative of $v\left(x ; x^{\prime}\right)$ are not independent because $D^{\mu} v=$ $D^{\mu} u$ and $D^{\prime \nu} v=-D^{\prime \nu} u$.) The second class of results is more involved and concerns the most general form for the vacuum polarization [9],

$$
i\left[{ }^{\mu} \Pi^{\nu}\right]\left(x ; x^{\prime}\right)=\sqrt{-g} \sqrt{-g^{\prime}} D_{\rho} D_{\sigma}^{\prime}\left[{ }^{\mu \rho} T^{\nu \sigma}\right]\left(x ; x^{\prime}\right),
$$

where $\left[{ }^{\mu \rho} T^{\nu \sigma}\right]\left(x ; x^{\prime}\right)=-\left[{ }^{\rho \mu} T^{\nu \sigma}\right]\left(x ; x^{\prime}\right)=-\left[{ }^{\mu \rho} T^{\sigma \nu}\right]\left(x ; x^{\prime}\right)=+\left[{ }^{\nu \sigma} T^{\mu \rho}\right]\left(x^{\prime} ; x\right)$.

The symmetries of cosmology — homogeneity and isotropy — do not reduce (13) to the form (9) . In addition to just $f_{1}(y, u, v)$ and $f_{2}(y, u, v)$, the anti-symmetric bi-tensor $\left[{ }^{\mu \rho} T^{\nu \sigma}\right]\left(x ; x^{\prime}\right)$ can involve three additional structure functions,

$$
\begin{aligned}
& {\left[\mu \rho T_{\nu \sigma}\right]=\partial_{\mu} \partial_{[\nu}^{\prime} y \partial_{\sigma]}^{\prime} \partial_{\rho} y \times f_{1}+\partial_{[\mu} y \partial_{\rho]} \partial_{[\nu}^{\prime} y \partial_{\sigma]}^{\prime} y \times f_{2}+\partial_{[\mu} y \partial_{\rho]} \partial_{[\nu}^{\prime} y \partial_{\sigma]}^{\prime} u \times f_{3}} \\
& +\partial_{[\mu} u \partial_{\rho]} \partial_{[\nu}^{\prime} y \partial_{\sigma]}^{\prime} y \times \tilde{f}_{3}+\partial_{[\mu} u \partial_{\rho]} \partial_{[\nu}^{\prime} y \partial_{\sigma]}^{\prime} u \times f_{4}+\partial_{[\mu} y \partial_{\rho]} u \partial_{[\nu}^{\prime} y \partial_{\sigma]}^{\prime} u \times f_{5}
\end{aligned}
$$

where $f_{i}=f_{i}(y, u, v)$ and $\tilde{f}_{3}(y, u, v)=f_{3}(y, u,-v)$, as dictated by symmetries of $\left[{ }_{\mu \rho} T_{\nu \sigma}\right]$. To see that only two of these structure functions are actually independent we first note that acting the derivatives in (13) results in only four algebraically independent tensors,

$$
\begin{aligned}
\left.D^{\rho} D^{\prime \sigma}{ }_{[\mu \rho} T_{\nu \sigma}\right]\left(x ; x^{\prime}\right)=\partial_{\mu} \partial_{\nu}^{\prime} y \times F_{1}+\partial_{\mu} y \partial_{\nu}^{\prime} y \times F_{2} \\
+\partial_{\mu} u \partial_{\nu}^{\prime} y \times F_{3}+\partial_{\mu} y \partial_{\nu}^{\prime} u \times \widetilde{F}_{3}+\partial_{\mu} u \partial_{\nu}^{\prime} u \times F_{4},
\end{aligned}
$$

where $F_{i}=F_{i}(y, u, v)(i=1,2,3,4)$ and $\tilde{F}_{3}(y, u, v)=F_{3}(y, u,-v)$. Furthermore, transversality implies two partial differential relations between the $F_{i}$, one proportional to the derivative of $y\left(x ; x^{\prime}\right)$ and the other proportional to the derivative of $u\left(x ; x^{\prime}\right)$ [16].

All of this suggests that there are only two master structure functions which we might call $\Phi=\Phi(y, u, v)$ and $\Psi=\Psi(y, u, v)$. In ref. [9] that suspicion was confirmed by demonstrating the transversality of the following 
substitutions for the coefficient functions $F_{i}(y, u, v)$,

$$
\begin{aligned}
F_{1} & =\left[-\left(4 y-y^{2}\right) \partial_{y}-(D-1)(2-y)-2(2-y) \partial_{u}+4 \cosh (v) \partial_{u}-4 \sinh (v) \partial_{v}\right] \Phi \\
F_{2} & =\left[(2-y) \partial_{y}-D+1-2 \partial_{u}\right] \Phi-\Psi, \\
F_{3} & =-2 e^{v}\left(\partial_{u}-\partial_{v}\right) \Phi-2 e^{v} \Psi+\Xi, \\
\widetilde{F}_{3} & =-2 e^{-v}\left(\partial_{u}+\partial_{v}\right) \Phi-2 e^{-v} \Psi+\Xi, \\
F_{4} & =-4 \Psi+(2-y) \Xi .
\end{aligned}
$$

Here the auxiliary function $\Xi(y, u, v)$ is,

$\Xi(y, u, v) \equiv\left(\partial_{u}^{2}-\partial_{v}^{2}\right) \int d y \Phi(y, u, v)+(2-y) \Psi(y, u, v)-(D-2) \int d y \Psi(y, u, v)$

Relations (16 20) can be inverted to obtain for the master structure functions,

$$
\begin{aligned}
{\left[(D-1)+2 \partial_{u}\right] \Phi } & =-\frac{1}{4}\left[(2-y) F_{1}+\left(4 y-y^{2}\right) F_{2}+(2-y)\left(F_{3}+\widetilde{F}_{3}\right)-F_{4}\right] \\
\sinh (v) \Psi & =\left[\cosh (v) \partial_{v}-\sinh (v) \partial_{u}\right] \Phi-\frac{1}{4}\left[F_{3}-\widetilde{F}_{3}\right]
\end{aligned}
$$

In the de Sitter invariant case only one de Sitter invariant master structure function suffices, that is $\Phi=\Phi(y)$ and $\Psi=0$.

On the other hand, one can relate the master structure functions to $f_{i}$ in (14). Below we quote separately the contributions from each of $f_{i}$ to master structure functions (this is denoted by the subscript $i=1,2,3,4,5$ 
on $\Phi_{i}$ and $\left.\Psi_{i}\right)$ :

$$
\begin{aligned}
\Phi_{1}= & \frac{H^{4}}{2}\left\{(D-1) f_{1}-(2-y) \partial_{y} f_{1}\right\} \\
\Psi_{1}= & \frac{H^{4}}{2}\left\{\left(\partial_{u}^{2}-\partial_{v}^{2}\right) f_{1}\right\} \\
\Phi_{2}= & \frac{H^{4}}{4}\left\{D(2-y) f_{2}+\left(4 y-y^{2}\right) \partial_{y} f_{2}\right\} \\
\Psi_{2}= & \frac{H^{4}}{4}\left\{(2-y)\left(\partial_{u}^{2}-\partial_{v}^{2}\right) f_{2}\right\} \\
\Phi_{3}= & \frac{H^{4}}{4}\left\{-(D-1)\left(f_{3}+\widetilde{f}_{3}\right)+(2-y) \partial_{y}\left(f_{3}+\widetilde{f}_{3}\right)-2 \partial_{y}\left(e^{v} f_{3}+e^{-v} \widetilde{f}_{3}\right)\right\} \\
\Psi_{3}= & \frac{H^{4}}{4}\left\{2 \partial_{y}\left[\partial_{u}\left(e^{v} f_{3}+e^{-v} \tilde{f}_{3}\right)-\partial_{v}\left(e^{v} f_{3}-e^{-v} \widetilde{f}_{3}\right)\right]-\left(\partial_{u}^{2}-\partial_{v}^{2}\right)\left(f_{3}+\widetilde{f}_{3}\right)\right\} \\
\Phi_{4}= & \frac{H^{4}}{4}\left\{\partial_{y} f_{4}\right\}, \\
\Psi_{4}= & \frac{H^{4}}{4}\left\{-(D-1) \partial_{y} f_{4}+(2-y) \partial_{y}^{2} f_{4}-2 \partial_{y} \partial_{u} f_{4}\right\} . \\
\Phi_{5}= & \frac{H^{4}}{4}\left\{-D f_{5}+2(2-y) \partial_{y} f_{5}-4 \cosh (v) \partial_{y} f_{5}\right\} \\
\Psi_{5}= & \frac{H^{4}}{4}\left\{(D-1) f_{5}-(D+1)(2-y) \partial_{y} f_{5}-\left(4 y-y^{2}\right) \partial_{y}^{2} f_{5}+2 \partial_{u} f_{5}\right. \\
& \left.+[-2(2-y)+4 \cosh (v)] \partial_{u} \partial_{y} f_{5}-4 \partial_{v} \partial_{y}\left[\sinh (v) f_{5}\right]-\left(\partial_{u}^{2}-\partial_{v}^{2}\right) f_{5}\right\} .
\end{aligned}
$$

These expressions also imply that the vacuum polarization can be described in terms of any two of the structure functions $f_{i}(y, u, v)$. When the result is de Sitter invariant then it requires only a single structure function, which can be either $f_{1}(y)$ or $f_{2}(y)$.

\section{The Derivation}

The first step in our analysis is to covariantize expression (5) by noting it can be written as,

$$
i\left[{ }^{\mu} \Pi^{\nu}\right]\left(x ; x^{\prime}\right)=2 \partial_{\alpha}^{\prime} \partial_{\beta}\left\{\frac{\sqrt{-g} \sqrt{-g^{\prime}}}{\left(a a^{\prime}\right)^{2}}\left[\eta^{\mu[\nu} \eta^{\alpha] \beta} \widehat{F}+\bar{\eta}^{\mu[\nu} \bar{\eta}^{\alpha] \beta} \widehat{G}\right]\right\}
$$


where we have rescaled $F\left(x ; x^{\prime}\right)$ and $G\left(x ; x^{\prime}\right)$ as

$$
F=\frac{\sqrt{-g} \sqrt{-g^{\prime}}}{\left(a a^{\prime}\right)^{2}} \widehat{F}, \quad G=\frac{\sqrt{-g} \sqrt{-g^{\prime}}}{\left(a a^{\prime}\right)^{2}} \widehat{G} .
$$

Now, when the measure factors $\sqrt{-g}$ and $\sqrt{-g^{\prime}}$ are pulled out of the curly brackets in (34), the external derivatives become covariant,

$$
i\left[{ }^{\mu} \Pi^{\nu}\right]\left(x ; x^{\prime}\right)=2 \sqrt{-g} \sqrt{-g^{\prime}} D_{\alpha}^{\prime} D_{\beta}\left\{\left(a a^{\prime}\right)^{-2}\left[\eta^{\mu[\nu} \eta^{\alpha] \beta} \widehat{F}+\bar{\eta}^{\mu[\nu} \bar{\eta}^{\alpha] \beta} \widehat{G}\right]\right\} .
$$

This follows from the observation that, because of the anti-symmetry in $\left.\mu \leftrightarrow \beta, D_{\beta}\left[{ }^{\mu \beta} T^{\nu \alpha}\right]=\partial_{\beta}{ }^{\mu \beta} T^{\nu \alpha}\right]+\Gamma_{\beta \sigma}^{\beta}\left[\mu \sigma T^{\nu \alpha}\right]$, where $\Gamma_{\beta \sigma}^{\beta}=(-g)^{-1 / 2} \partial_{\sigma}(-g)^{1 / 2}$. The analogous identity holds for the $D_{\alpha}^{\prime}$ derivative.

The next step is to put the terms involving $\eta^{\mu \nu} / a a^{\prime}$ and $\bar{\eta}^{\mu \nu} / a a^{\prime}$ in (36) into a covariant form using relations (11,12). Upon inserting these relations into (36), and then comparing with Eq. (14), we find,

$$
\begin{aligned}
f_{1} & =\frac{1}{2 H^{4}}(\widehat{F}+\widehat{G}), \quad f_{2}=0, \\
f_{3} & =\frac{1}{H^{4}}(\widehat{F}+\widehat{G})=\tilde{f}_{3}, \\
f_{4} & =\frac{1}{H^{4}}(-y \widehat{F}+(2-y) \widehat{G}), \quad f_{5}=-\frac{1}{H^{4}}(\widehat{F}+\widehat{G}) .
\end{aligned}
$$

The third step is to insert relations (37) into the master structure functions Eqs. (24 33) for each of the five structure functions $f_{i}$, and then summing to find,

$$
\begin{aligned}
\Phi & =\sum_{i=1}^{5} \Phi_{i}=-\frac{1}{2} \partial_{y} F \\
\Psi & =\sum_{i=1}^{5} \Psi_{i}=\frac{1}{2}\left[(D-1)+y \partial_{y}+2 \partial_{u}\right] \partial_{y} F+\partial_{y}^{2} G \\
& =-\left[(D-1)-(2-y) \partial_{y}+2 \partial_{u}\right] \Phi+\partial_{y}^{2}(F+G) .
\end{aligned}
$$

This implies that we can express the vacuum polarization tensor in terms of the two structure functions $f_{1}$ and $f_{2}$ as in Eq. (9). Indeed, with the help of Eqs. (24 27) we get:

$$
\begin{aligned}
\Phi_{1}+\Phi_{2} & =\frac{H^{4}}{4}\left(\partial_{u}^{2}-\partial_{v}^{2}\right) g_{1}, \quad g_{1}=2 f_{1}+(2-y) f_{2} \\
\Psi_{1}+\Psi_{2} & =\frac{H^{4}}{4}\left\{\left[(D-1)-(2-y) \partial_{y}\right] g_{1}+4 \partial_{y} f_{2}\right\} .
\end{aligned}
$$


These equations can be solved for $g_{1}$ and $f_{2,1}$ in terms of $\Phi \equiv \Phi_{1}+\Phi_{2}$ and $\Psi \equiv \Psi_{1}+\Psi_{2}$. Using the 'light-cone' coordinates, $x_{ \pm}=(u \pm v) / 2$ $\left(x_{+}=\ln (a), x_{-}=\ln \left(a^{\prime}\right)\right)$, and requiring interchange symmetry gives,

$$
\begin{aligned}
g_{1}(y, u, v) & =\int_{0}^{x_{+}} d \bar{x}_{+} \int_{0}^{x_{-}} d \bar{x}_{-} \Psi\left(y, \bar{x}_{+}+\bar{x}_{-}, \bar{x}_{+}-\bar{x}_{-}\right) \\
f_{2} & =\frac{2-y}{4} g_{1}+\int d y^{\prime}\left[\frac{1}{H^{4}} \Phi\left(y^{\prime}, u, v\right)-\frac{D-2}{4} g_{1}\left(y^{\prime}, u, v\right)\right]+\beta(u, v), \\
f_{1} & =\frac{1}{2}\left[g_{1}-(2-y) f_{2}\right]
\end{aligned}
$$

where $\alpha\left(y, x_{ \pm}\right)$and $\beta(u, v)$ are arbitrary functions, and $\Psi$ and $\Phi$ are given in Eqs. (38 39). Together with Eqs. (35), these expressions provide the desired procedure for transforming the vacuum polarization tensor from the form given in (5) into (9-13), and thus constitute the main result of this paper.

In the subsequent section we show that these transformations indeed work for the vacuum polarization induced by the one loop vacuum fluctuations of a minimally coupled massless scalar on de Sitter background, providing a nontrivial check of Eqs. (38 41).

\section{Correspondence}

Here we consider the vacuum polarization induced by one loop vacuum fluctuations of a massless minimally coupled scalar on de Sitter background.

If we call the scalar propagator $i \Delta\left(x ; x^{\prime}\right)$ then the one loop contribution to the vacuum polarization is of the form,

$$
\begin{aligned}
& i\left[{ }^{\mu} \Pi^{\nu}\right]\left(x ; x^{\prime}\right)=-2 i e^{2} \sqrt{-g} g^{\mu \nu} i \Delta(x ; x) \delta^{D}\left(x-x^{\prime}\right) \\
& +2 e^{2} \sqrt{-g} g^{\mu \rho} \sqrt{-g^{\prime}} g^{\prime \nu \sigma}\left[\partial_{\rho} i \Delta\left(x ; x^{\prime}\right) \partial_{\sigma}^{\prime} i \Delta\left(x ; x^{\prime}\right)-i \Delta\left(x ; x^{\prime}\right) \partial_{\rho} \partial_{\sigma}^{\prime} i \Delta\left(x ; x^{\prime}\right)\right] \\
& +i \delta Z \partial_{\rho}\left[\sqrt{-g}\left(g^{\mu \nu} g^{\rho \sigma}-g^{\mu \sigma} g^{\nu \rho}\right) \partial_{\sigma} \delta^{D}\left(x-x^{\prime}\right)\right],
\end{aligned}
$$

were $\delta Z$ is a counterterm, and $e$ is the electromagnetic coupling $\left(e^{2} /(4 \pi) \equiv\right.$ $\alpha_{e} \simeq 1 / 137$ ). For the special case of the massless, minimally coupled scalar the propagator obeys

$$
\sqrt{-g} \square i \Delta_{A}\left(x ; x^{\prime}\right)=i \delta^{D}\left(x-x^{\prime}\right),
$$


where $\square \equiv(-g)^{-\frac{1}{2}} \partial_{\mu}\left(\sqrt{-g} g^{\mu \nu} \partial_{\nu}\right)$ is the covariant d'Alembertian as it acts on a (bi-)scalar function. The unique solution that preserves the symmetries of the cosmological background (homogeneity, isotropy and spatial flatness) can be written as a sum of a de Sitter invariant part and a de Sitter breaking part $\left(\propto u=\ln \left(a a^{\prime}\right)\right)$ as [21, 22]:

$$
i \Delta_{A}\left(x ; x^{\prime}\right)=A\left(y\left(x ; x^{\prime}\right)\right)+k u,
$$

where the constant $k$ is

$$
k=\frac{H^{D-2}}{(4 \pi)^{\frac{D}{2}}} \frac{\Gamma(D-1)}{\Gamma\left(\frac{D}{2}\right)}
$$

and the de Sitter invariant part of the propagator $A(y)$ obeys - as a consequence of the propagator equation (43) - the equation

$$
\left(4 y-y^{2}\right) A^{\prime \prime}(y)+D(2-y) A^{\prime}(y)=(D-1) k .
$$

The solution of this equation can be written as a combination of two Gauss' hypergeometric functions. Upon inserting these into (42), one obtains for the vacuum polarization

$$
\begin{aligned}
& i\left[{ }^{\mu} \Pi^{\nu}\right]\left(x ; x^{\prime}\right)=2 e^{2} \sqrt{-g} g^{\mu \rho} \sqrt{-g^{\prime}} g^{\prime \nu \sigma}\left\{\partial_{\rho} y \partial_{\sigma}^{\prime} y \times\left(A^{\prime 2}-A A^{\prime \prime}-k u A^{\prime \prime}\right)\right. \\
& \left.-\partial_{\rho} \partial_{\sigma}^{\prime} y \times\left(A A^{\prime}+k u A^{\prime}\right)+\partial_{\rho} u \partial_{\sigma}^{\prime} y \times k A^{\prime}+\partial_{\rho} y \partial_{\sigma}^{\prime} u \times k A^{\prime}+\partial_{\rho} u \partial_{\sigma}^{\prime} u \times k^{2}\right\}
\end{aligned}
$$

plus local terms $\propto \delta^{D}\left(x-x^{\prime}\right)$, which are not relevant for this consideration (see e.g. Ref. [9]).

In order to establish correspondence, it suffices to consider two independent components of $i\left[{ }^{\mu} \Pi^{\nu}\right]$ in (47). It turns out that taking the 00 and $i j$ $(i \neq j)$ components of (47) leads to rather simple expressions,

$$
\begin{gathered}
i\left[{ }^{0} \Pi^{0}\right]\left(x ; x^{\prime}\right)=2 e^{2} \sqrt{-g} \sqrt{-g^{\prime}} \frac{H^{2}}{a a^{\prime}} \\
\times\left\{\left[4+(2-y)^{2}-4(2-y) \cosh (v)\right]\left(A^{2}-A A^{\prime \prime}-k u A^{\prime \prime}\right)\right. \\
\left.+[(2-y)-4 \cosh (v)]\left(A A^{\prime}+k u A^{\prime}\right)+[-2(2-y)+4 \cosh (v)] \times k A^{\prime}+k^{2}\right\} \\
\left.i\left[{ }^{i} \Pi^{j}\right]\left(x ; x^{\prime}\right)\right|_{i \neq j}=2 e^{2} \sqrt{-g} \sqrt{-g^{\prime}} \frac{H^{2}}{a a^{\prime}}\left\{\left[-4 a \Delta x^{i} a^{\prime} \Delta x^{j}\right]\left(A^{\prime 2}-A A^{\prime \prime}-k u A^{\prime \prime}\right) .\right.
\end{gathered}
$$


On the other hand, from Eq. (15) we read off,

$$
\begin{aligned}
i\left[{ }^{0} \Pi^{0}\right] & =-\nabla^{\prime} \cdot \nabla F \\
& =2 \sqrt{-g} \sqrt{-g^{\prime}} \frac{H^{2}}{a a^{\prime}}\left[(D-1)-2(2-y) \partial_{y}+4 \cosh (v) \partial_{y}\right] \partial_{y} \widehat{F}(50) \\
i\left[{ }^{i} \Pi^{j}\right]_{i \neq j} & =-\partial_{i}^{\prime} \partial_{j}(F+G) \\
& =\sqrt{-g} \sqrt{-g^{\prime}} \frac{H^{2}}{a a^{\prime}}\left\{\left[4 a \Delta x^{i} a^{\prime} \Delta x^{j}\right] \partial_{y}^{2}(\widehat{F}+\widehat{G})\right\}
\end{aligned}
$$

We see from Eq. (48) that $i\left[{ }^{0} \Pi^{0}\right]$ contains dependencies in the form: (a) a function of $y$, (b) a function of $y$ times $u$ and (c) a function of $y$ times $\cosh (v)$. On the other hand, inspecting (150) and assuming that $\widehat{F}$ does not depend on $v$ (this in fact follows from the spatial diagonal components $i\left[{ }^{i} \Pi^{i}\right]$ ) tells us that the following equations must be separately satisfied,

$$
\begin{aligned}
& e^{2}\left\{-2(2-y)\left(A^{\prime 2}-A A^{\prime \prime}-k u A^{\prime \prime}\right)-2(A+k u) A^{\prime}+2 k A^{\prime}\right\}=2 \partial_{y}^{2} \widehat{F} \\
& e^{2}\left\{\left[4+(2-y)^{2}\right]\left(A^{\prime 2}-A A^{\prime \prime}-k u A^{\prime \prime}\right)\right. \\
& \left.\quad+2(2-y)(A+k u) A^{\prime}-2(2-y) k A^{\prime}+k^{2}\right\}=\left[(D-1)-2(2-y) \partial_{y}\right] \partial_{y} \widehat{F} .
\end{aligned}
$$

Multiplying the first equation by $(2-y)$ and adding the second results in,

$$
\begin{array}{r}
\left.e^{2}\left\{\left(4 y-y^{2}\right) A^{\prime 2}+(D-1)(2-y)(A+k u) A^{\prime}-k(D-1)(A+k u)+k^{2}\right)\right\} \\
=(D-1) \partial_{y} \widehat{F},
\end{array}
$$

where we also made use of Eq. (46) . Now, multiplying this by $-1 /[2(D-1)]$ and separating the $u$-dependent terms yields

$$
\begin{aligned}
-\frac{1}{2} \partial_{y} \widehat{F}= & -\frac{e^{2}}{2(D-1)}\left\{(D-1)\left[(2-y) A^{\prime}-k\right] A+\left(4 y-y^{2}\right) A^{\prime 2}+k^{2}\right\} \\
& -\frac{e^{2} k}{2}\left[(2-y) A^{\prime}-k\right] u=\Phi
\end{aligned}
$$

where the last equality follows from (38). This equation agrees with Eq. (44) in Ref. [9], establishing the first part of the correspondence for the minimally coupled massless scalar field. 
Next we consider Eqs. (49) and (51), which give us information about the second structure function $G$,

$$
-2 e^{2}\left(A^{\prime 2}-A A^{\prime \prime}-k u A^{\prime \prime}\right)=\partial_{y}^{2}(\widehat{F}+\widehat{G}) .
$$

In order to get the second master structure function (38), we add

$$
\begin{aligned}
-\left[(D-1)-(2-y) \partial_{y}+2 \partial_{u}\right] \Phi= & 2 e^{2}\left(A^{\prime 2}-A A^{\prime \prime}-k u A^{\prime \prime}\right) \\
& +\frac{e^{2} k}{2}\left[(2-y) A^{\prime}-k\right]
\end{aligned}
$$

to both sides of Eq. (53). This procedure results in

$$
\Psi=\frac{e^{2} k}{2}\left[(2-y) A^{\prime}-k\right]
$$

and agrees with Eq. (45) of Ref. [9]. This establishes the second part of the correspondence for the minimally coupled massless scalar field, and completes our (nontrivial) check of the main results (38 41).

\section{Discussion}

We have established how to transform from the non-covariant form (5) of the vacuum polarization tensor used in Refs. [4, 5, 6, 7, to the covariant form (9) introduced in our recent work [9]. Our main results are the transformation formulae (38 41), based on which one can effortlessly move from one representation of the vacuum polarization to another. Our results can be useful for studies of the one loop vacuum polarization induced by gravitons, scalars and fermions on a locally de Sitter background [10]. In particular, it provides a way of characterizing the irreducible effect on photons of de Sitter breaking in the gravitational sector. An important technical result of some importance is the improved procedure (50,51) for extracting the noncovariant structure functions $F(x ; x)$ and $G\left(x ; x^{\prime}\right)$.

Our results might be useful for understanding the structure of the photon vacuum polarization tensor on arbitrary background geometries. Namely, on de Sitter background the vacuum polarization is given in terms of two biscalar structure functions which generally violate de Sitter symmetry. In the special case, when the de Sitter symmetry is unbroken, one (de Sitter invariant) structure function suffices to fully specify the vacuum polarization 
tensor. It is tempting to try generalizing our results (9) to generic backgrounds by replacing the de Sitter invariant length $y\left(x ; x^{\prime}\right)$ by the appropriate geodesic distance $\ell\left(x ; x^{\prime}\right)$, in which case (9) would become

$$
\left[{ }_{\mu \rho} T_{\nu \sigma}\right]\left(x ; x^{\prime}\right)=\partial_{\mu} \partial_{[\nu}^{\prime} \ell^{2} \partial_{\sigma]}^{\prime} \partial_{\rho} \ell^{2} \times f_{1}+\partial_{[\mu} \ell^{2} \partial_{\rho]} \partial_{[\nu}^{\prime} \ell^{2} \partial_{\sigma]}^{\prime} \ell^{2} \times f_{2} .
$$

One would think there must be at least two structure functions in a general metric because photons have two polarizations and birefringence is known to occur in some backgrounds [33]. On the other hand, there cannot be more than six structure functions because that is the number of independent components of a transverse bi-vector density. It would be interesting to see if tighter bounds can be derived.

\section{Acknowledgements}

This work was partially supported by NSF grant PHY-1205591, and by the Institute for Fundamental Theory at the University of Florida.

\section{References}

[1] M. E. Peskin and D. V. Schroeder, Reading, USA: Addison-Wesley (1995) $842 \mathrm{p}$.

[2] K. E. Leonard and R. P. Woodard, Phys. Rev. D 85, 104048 (2012) arXiv:1202.5800 [gr-qc]].

[3] A. Marunovic and T. Prokopec, arXiv:1209.4779 [hep-th].

[4] T. Prokopec, O. Tornkvist and R. P. Woodard, Phys. Rev. Lett. 89, 101301 (2002) astro-ph/0205331].

[5] T. Prokopec, O. Tornkvist and R. P. Woodard, Annals Phys. 303, 251 (2003) gr-qc/0205130.

[6] T. Prokopec and R. P. Woodard, Annals Phys. 312, 1 (2004) gr-qc/0310056.

[7] T. Prokopec and E. Puchwein, JCAP 0404, 007 (2004) [astro-ph/0312274]. 
[8] H. Degueldre and R. P. Woodard, "One Loop Field Strengths of Charges and Dipoles on a Locally de Sitter Background," in preparation (2012).

[9] K. E. Leonard, T. Prokopec and R. P. Woodard, "Covariant Vacuum Polarizations on de Sitter Background," arXiv:1210.6968 [gr-qc].

[10] K. E. Leonard and R. P. Woodard, "The One Loop Vacuum Polarization from Inflationary Gravitons," in preparation (2012).

[11] S. -P. Miao and R. P. Woodard, Class. Quant. Grav. 23, 1721 (2006) gr-qc/0511140].

[12] S. P. Miao and R. P. Woodard, Phys. Rev. D 74, 024021 (2006) gr-qc/0603135.

[13] S. -P. Miao, arXiv:0705.0767 [hep-th].

[14] S. P. Miao, arXiv:1207.5241 [gr-qc].

[15] S. -P. Miao and R. P. Woodard, Class. Quant. Grav. 25, 145009 (2008) arXiv:0803.2377 [gr-qc]].

[16] S. P. Miao, N. C. Tsamis and R. P. Woodard, J. Math. Phys. 51, 072503 (2010) [arXiv:1002.4037 [gr-qc]].

[17] T. Prokopec and R. P. Woodard, "Vacuum polarization and photon mass in inflation," Am. J. Phys. 72 (2004) 60 astro-ph/0303358.

[18] A. Higuchi, D. Marolf and I. A. Morrison, Class. Quant. Grav. 28, 245012 (2011) [arXiv:1107.2712 [hep-th]].

[19] S. P. Miao, N. C. Tsamis and R. P. Woodard, Class. Quant. Grav. 28, 245013 (2011) arXiv:1107.4733 [gr-qc]].

[20] B. Allen and A. Folacci, Phys. Rev. D 35, 3771 (1987).

[21] V. K. Onemli and R. P. Woodard, Class. Quant. Grav. 19, 4607 (2002) gr-qc/0204065].

[22] V. K. Onemli and R. P. Woodard, Phys. Rev. D 70, 107301 (2004) gr-qc/0406098]. 
[23] S. Park and R. P. Woodard, Phys. Rev. D 83, 084049 (2011) arXiv:1101.5804 [gr-qc]].

[24] S. Park and R. P. Woodard, Phys. Rev. D 84, 124058 (2011) arXiv:1109.4187 [gr-qc]].

[25] E. O. Kahya, V. K. Onemli and R. P. Woodard, Phys. Rev. D 81, 023508 (2010) [arXiv:0904.4811 [gr-qc]].

[26] N. C. Tsamis and R. P. Woodard, J. Math. Phys. 48, 052306 (2007) gr-qc/0608069.

[27] N. C. Tsamis and R. P. Woodard, Commun. Math. Phys. 162, 217 (1994).

[28] S. P. Miao, N. C. Tsamis and R. P. Woodard, J. Math. Phys. 50, 122502 (2009) [arXiv:0907.4930 [gr-qc]].

[29] S. P. Miao, N. C. Tsamis and R. P. Woodard, J. Math. Phys. 52, 122301 (2011) [arXiv:1106.0925 [gr-qc]].

[30] E. O. Kahya, S. P. Miao and R. P. Woodard, J. Math. Phys. 53, 022304 (2012) [arXiv:1112.4420 [gr-qc]].

[31] P. J. Mora, N. C. Tsamis and R. P. Woodard, arXiv:1205.4468 [gr-qc].

[32] P. J. Mora and R. P. Woodard, Phys. Rev. D 85, 124048 (2012) arXiv:1202.0999 [gr-qc]].

[33] B. L. Hu and K. Shiokawa, Phys. Rev. D 57, 3474 (1998) gr-qc/9708023]. 